\title{
AN OVERVIEW ON ASHWAGANDHA: A RASAYANA (REJUVENATOR) OF AYURVEDA
}

\author{
Narendra Singh", Mohit Bhalla, Prashanti de Jager* and Marilena Gilca** \\ International Institute of Herbal Medicine (IIHM), Gomtinagar, Lucknow - 226010, INDIA \\ *Ayurvedic Scholar at the International Institute of Herbal Medicine (IIHM), Lucknow, India, \\ **Biochemistry Department, Faculty of General Medicine, "Carol Davila" University of Medicine and \\ Pharmaceutics, Bucharest, Romania, Visiting Scientist at the International Institute of Herbal Medicine \\ (IIHM), Lucknow, India \\ *Email: drnarendrasingh@gmail.com
}

\begin{abstract}
Withania somnifera (Ashawagandha) is very revered herb of the Indian Ayurvedic system of medicine as a Rasayana (tonic). It is used for various kinds of disease processes and specially as a nervine tonic. Considering these facts many scientific studies were carried out and its adaptogenic / anti-stress activities were studied in detail. In experimental models it increases the stamina of rats during swimming endurance test and prevented adrenal gland changes of ascorbic acid and cortisol content produce by swimming stress. Pretreatment with Withania somnifera (WS) showed significance protection against stress induced gastric ulcers. WS have anti-tumor effect on Chinese Hamster Ovary (CHO) cell carcinoma. It was also found effective against urethane induced lung-adenoma in mice. In some cases of uterine fibroids, dermatosarcoma, long term treatment with WS controlled the condition. It has a Cognition Promoting Effect and was useful in children with memory deficit and in old age people loss of memory. It was also found useful in neurodegenerative diseases such as Parkinson's, Huntington's and Alzeimer's diseases. It has GABA mimetic effect and was shown to promote formation of dendrites. It has anxiolytic effect and improves energy levels and mitochondrial health. It is an anti-inflammatory and antiarthritic agent and was found useful in clinical cases of Rheumatoid and Osteoarthritis. Large scale studies are needed to prove its clinical efficacy in stress related disorders, neuronal disorders and cancers.
\end{abstract}

Key words: Withania somnifera, rejuvenator, adaptogen / anti-stress, anti-tumor, neuroregenerative, anti-arthritic.

\section{Introduction}

Ashwagandha (Withania somnifera, fam. Solanaceae) is commonly known as "Indian Winter cherry" or "Indian Ginseng". It is one of the most important herb of Ayurveda (the traditional system of medicine in India) used for millennia as a Rasayana for its wide ranging health benefits. Rasayana is described as an herbal or metallic preparation that promotes a youthful state of physical and mental health and expands happiness. These types of remedies are given to small children as tonics, and are also taken by the middle-aged and elderly to increase longevity. Among the ayurvedic Rasayana herbs, Ashwagandha holds the most prominent place. It is known as "Sattvic Kapha Rasayana" Herb (Changhadi, 1938). Most of the Rasayana herbs are adaptogen / anti-stress agents.

Ashwagandha is commonly available as a churna, a fine sieved powder that can be mixed with water, ghee (clarified butter) or honey. It enhances the function of the brain and nervous system and improves the memory. It improves the function of the reproductive system promoting a healthy sexual and reproductive balance. Being a powerful adaptogen, it enhances the body's resilience to stress. Ashwagandha improves the body's defense against disease by improving the cell-mediated immunity. It also possesses potent antioxidant properties that help protect against cellular damage caused by free radicals.

\section{Chemical Composition}

iThe biologically active chemical constituents of Withania somnifera (WS) include alkaloids (isopelletierine, anaferine, cuseohygrine, anahygrine, etc.), steroidal lactones (withanolides, withaferins) and saponins (Mishra, 2000 et al., 2000). Sitoindosides and acylsterylglucosides in Ashwagandha are anti-stress agents. Active principles of Ashwagandha, for instance the sitoindosides VII-X and Withaferin-A, have been shown to have significant anti-stress activity against acute models of experimental stress (Bhattacharya et al., 1987). Many of its constituents support immunomodulatory actions (Ghosal et al., 1989). The aerial parts of Withania somnifera yielded 5-dehydroxy withanolide-R and withasomniferin-A (Atta-urRahman et al., 1991).

\section{Classical Uses of Ashwagandha}

Ayurveda, the traditional system of medicine practiced in India can be traced back to 6000 BC (Charak Samhita, 1949). For most of these 6000 years Ashwagandha has been used as a Rasayana. The root of Ashwagandha is regarded as tonic, aphrodisiac, narcotic, diuretic, anthelmintic, astringent, thermogenic and stimulant. The root smells like horse 
("ashwa"), that is why it is called Ashwagandha (on consuming it gives the power of a horse). It is commonly used in emaciation of children (when given with milk, it is the best tonic for children), debility from old age, rheumatism, vitiated conditions of vata, leucoderma, constipation, insomnia, nervous breakdown, goiter etc. (Sharma, 1999). The paste formed when roots are crushed with water is applied to reduce the inflammation at the joints (Bhandari, 1970). It is also locally applied in carbuncles, ulcers and painful swellings (Kritikar and Basu, 1935). The root in combination with other drugs is prescribed for snake venom as well as in scorpion-sting. It also helps in leucorrhoea, boils, pimples, flatulent colic, worms and piles (Misra, 2004). The Nagori Ashwagandha is the supreme among all Ashwagandha varieties. Maximum benefit appears when fresh Ashwagandha powder is used (Singh, 1983).

The leaves are bitter and are recommended in fever, painful swellings. The flowers are astringent, depurative, diuretic and aphrodisiac. The seeds are anthelmintic and combined with astringent and rock salt remove white spots from the cornea. Ashwagandharishta prepared from it is used in hysteria, anxiety, memory loss, syncope, etc. It also acts as a stimulant and increases the sperm count (Sharma, 1938).

\section{Scientific Studies on Ashwagandha \\ Adaptogenic / Anti-stress effect}

Aswagandha is compared well with Eleutherococcus senticosus (Siberian Ginseng) and Panax Ginseng (Chinese / Korean Ginseng) in its adaptogenic properties, and hence it is popularly known as Indian Ginseng (Singh et al., 2010). The extensive studies on the biological model of animals for the adaptogenic / anti-stress properties of Ashwagandha (Abbas and Singh, 2006; Kalsi et al., 1987; Singh et al., 1976, 1977, 1981, 1982, 1993a, 1993b, 2003; (Singh, 1995a, 1995b, 2006, 2008) have shown it to be effective in increasing the stamina (physical endurance) and preventing stress induced gastric ulcer, carbon tetrachloride $\left(\mathrm{CCl}_{4}\right)$ induced hepatotoxicity and mortality. Ashawagandha have similar anti-stress activity in rats (Archana \& Namasivayam, 1999). An aqueous suspension of Ashwagandha root was used at $100 \mathrm{mg} / \mathrm{kg} / \mathrm{oral}$ dosage. The results indicate a significant increase in the plasma corticosterone level, phagocytic index and avidity index in rats subjected to cold swimming stress. In the rats pretreated with the drug, these parameters were near control values and an increase in the swimming time was observed. These results indicate that Withania somnifera used in the crude form is a potent anti-stress agent. The results of above studies lend support to the hypothesis of tonics, vitalizers and rejuvenators of Ayurveda which indicate clinical use of Withania somnifera in the prevention and treatment of many stress induced diseases like arteriosclerosis, premature ageing, arthritis, diabetes, hypertension and malignancy (Singh, 1986, 2005; Singh and Misra, 1993).

\section{i. Effect on swimming performance}

Ashwagandha was shown to increase swimming performance in rats as judged by increase in swimming time during physical endurance test. Ashwagandha's antistress properties have been investigated in all these studies using adult rats were carried out by swimming endurance stress test. Ashwagandha treated animals showed a significant increase in the duration of swimming time as compared to control. The control group of mice swam for a mean time of 385 minutes, whereas the drugtreated animals continued to swim for a mean duration of 740 minutes. Thus, the swimming time was approximately doubled after Withania somnifera (WS) treatment.

\section{ii. Effect on cortisol and ascorbic acid contents of adrenals}

The cortisol content of adrenals was reduced significantly in animals subjected to $5 \mathrm{~h}$ constant swimming as compared to non-swimmer group. Pretreatment with WS prevented reduction of the cortisol content of adrenals. The ascorbic acid content was also reduced significantly after $5 \mathrm{~h}$ of swimming as compared to the animal of non-swimmer group. Pretreatment with WS prevent reduction in ascorbic acid content which occurs after swimming stress. Thus, Withania somnifera treatment prevents, decrease of adrenal cortisol and ascorbic acid which occurs due to swimming stress.

\section{iii. Anti-ulcerogenic effect}

Ashwagandha was found to be useful in the prevention of stress-induced ulcers of the gastrointestinal tract (Singh et al. 1982). It showed significant protection against $18 \mathrm{~h}$ immobilization, cold + immobilization (4h) and aspirin induced gastric ulcers and lowered the mean ulcer index in rats.

\section{iv. Effect on leucocytosis}

Ashwagandha given to a group of mice with milk injection produced reduction in leucocytosis.

\section{v. Anabolic effects:}

There was a significant increase in the body weights of the Ashwagandha treated group as compared to control for a period of 3 months in rats.

\section{Acute toxicity studies}

In acute toxicity studies the $\mathrm{LD}_{50}$ of Withania somnifera was found to be $1750 \mathrm{mg}$ (p.o.) in albino mice. 


\author{
Anti-tumor effect \\ Effect on Chinese Hamster Ovary (CHO) cells carcinoma
}

Withania roots caused the inhibitory effect of about $49 \%$ on colony forming efficiency of CHO cells. It inhibits the cell growth and prevents the cell attachment. It induced long term growth inhibition of CHO cells which was dependent on the cell density and duration of Ashwagandha exposure (Sumantran et al., 2007). This knowledge in turn will assist oncologists who plan to use the Ashwagandha as 'synergizers with conventional chemotherapy or radiation therapy.

\title{
Effect on Urethane induced lung-adenoma in mice and other studies:
}

Ashwagandha was found to be very useful in experimental carcinogenesis in the crude form. It prevented urethaneinduced lung-adenomas in mice. The other effects of urethane like leucopoenia were also prevented. Urethane, which is a chemical stressor, causes variety of ill effects, all of which were prevented by Withania. The drug can be used as an adjunct to cancer chemotherapy or radiotherapy. Besides having an anti-cancer effect it will also reduce the side effects of anti-cancer agents, which invariably reduce immunity and quality of life. WS also acts as an immunomodulator and hence can enhance life span of cancer patients, where lowered immunity states of the patient are the cause of concern. Our results suggest its use as anti-tumor (Singh et al. 1979, 1981, 1986, 2010) and immunomodulator agent (Dixit et al., 1995).

The research and studies of Ashwagandha's activities in the inhibition and reduction of tumour growth have shown encouraging evidence that this remarkable herb may prove to be extremely effective in the treatment of tumor type diseases including cancer (Singh and Gilca, 2010). It also improves the white cell count (WBC) and function, which are depleted in the chemotherapeutic treatment of cancer. Ashwagandha in the treatment of fibroid tumors of the uterus showed reduction of uterine bleeding tendencies and disappearance of fibroids after long treatment (Abbas et al. 2004, 2005).

\section{Effect on Central Nervous System Cognition Promoting Effect}

Ashwagandha is a well known Ayurvedic Rasayana, and belongs to a sub-group of Rasayanas known as Medhyarasayanas. Medhya typically refers to the mind and mental/intellectual capacity. Thus, Medhya Rasayana like Ashwagandha, is used to promote intellect and memory. The cognition-promoting effect of Medhya Rasayanas is best seen in children with memory deficits, or when memory is compromised following head injury, or a prolonged illness and in old age (Singh and Udupa., 1993).

\section{Effect on neurodegenerative diseases such as Parkinson's, Huntington's and Alzheimer's diseases}

In patients with Alzheimer's disease, neuritic atrophy and synaptic loss (Dickon and Vicker, 2001) are considered the major causes of cognitive impairment, as based on the results of neuropathological post-mortem studies of the brain (Dekosky \& Scheff, 1990). In the brains of patients suffering from other neurodegenerative diseases such as Parkinson's disease, Huntington's disease, and Creutzfeldt- Jakob disease, the atrophy of neurites has also been observed as a significant part of the etiology. There are dozens of studies that show that Ashwagandha slows, stops, reverses or removes neuritic atrophy and synaptic loss. Therefore Ashwagandha can be used to treat Alzheimer's, Parkinson's, Huntington's and other neurodegenerative diseases at any stage of the disease, even before a person has been diagnosed and is still in the state of mild forgetfulness, etc. Glycowithanolides withaferin- A and sitoindosides VII-X isolated from the roots of Ashwagandha significantly reversed ibotenic acid induced cognitive defects in Alzheimer's disease model (Bhattacharya et al., 1995).

Ashwagandha has been described as a nervine tonic (Singh et al., 1988, 1993) in Ayurveda and that is why it is a common ingredient of Ayurvedic tonic. Tonics, rejuvenators and vitalizers of Ayurveda appear to allay disease and induce immunity (Singh et al., 1986) and longevity in the users.

Pretreatment with Ashwaganda extract was found to prevent all the changes in antioxidant enzyme activities, catecholamine content, dopaminergic D2 receptor binding and tyrosine hydroxylase expression induced by 6hydroxydopamine (6-OHDA) in rats (an animal model of Parkinson's disease) in a dose-dependent manner. Thus, these results suggest that Ashwagandha may be helpful in protecting the neuronal injury in Parkinson's disease (Nagashyana et al., 2000).

\section{GABA-mimetic effect on neurodegeneration and neuroregenerative potential}

Behavioral experiments have lent support to the GABA-mimetic activity of Ashwagandha root extract. GABAergic neurodegeneration due to neuroleptic-induced excitotoxicity and oxidative stress is one of the etiopathological mechanisms in the pathophysiology of tardive dyskinesia (Gunne et al., 1993) and GABA agonists are shown to be effective in ameliorating the symptoms of tardive dyskinesia. The beneficial effect of Ashwagandha root extract might be due to its GABA mimetic activity. Ashwagandha, its constituents and the metabolites of its constituents promote the growth of nerves after taking it for 7 days.

An intriguing study demonstrated that chronic oral administration of withanoside IV attenuated the axonal, dendritic and synaptic losses and memory deficits induced by amyloid peptide $A \beta(25-35)$ in mice (Kuboyama et al, 2006). After oral administration in mice, withanoside IV was metabolized into sominone, which induced marked recovery in neurites and synapses and also enhanced axonal and dendritic outgrowth and synaptogenesis. These effects were maintained for at least 7 days after discontinuing withanoside IV administration. These data suggest that withanoside IV, and its metabolite, sominone, may have clinical usefulness as antidementia drugs.

Another team found that the methanol extract of Ashwagandha $(5 \mathrm{mg} / \mathrm{ml})$ significantly increased the percentage of 
cells with neurites in human neuroblastoma SK-N-SH cells. The effect of the extract was dose-and time-dependent. mRNA levels of the dendritic markers MAP2 and PSD-95 by RT-PCR were found to be markedly increased by treatment with the extract. Immunocytochemistry demonstrated the specific expression of MAP2 in neurites extended by the extract. These results suggest that the methanol extract of Ashwagandha promotes the formation of dendrites (Kulkarni et al., 1993).

\section{Anxiolytic effect:}

Ashwagandha induced a calming anxiolytic effect that was comparable to the drug Lorazepam in all three standard Anxiety tests: the elevated plus-maze, social interaction and the feeding latency in an unfamiliar environment. Further, both Ashwagandha and Lorazepam, reduced rat brain levels of tribulin, an endocoid marker of clinical anxiety, when the levels were increased following administration of the anxiogenic agent, pentylenetetrazole.

Ashwagandha also exhibited an antidepressant effect, comparable with that induced by imipramine, in two standard tests, the forced swim-induced 'behavioral despair' and 'learned helplessness' tests. The investigations support the use of Ashwagandha as a mood stabilizer in clinical conditions of anxiety and depression. (Abdel-Magied et al., 2001)

\section{Effect on Energy levels and Mitochondrial Health:}

The effect of Ashwagandha on glycosaminoglycan synthesis in the granulation tissue of carrageenin-induced air pouch granuloma was studied. Ashwagandha is shown to exert significant inhibitory effect on incorporation of ribosome $-35 \mathrm{~S}$ into the granulation tissue. The uncoupling effect on oxidative phosphorylation (ADP/O ratio reduction) was also observed in the mitochondria of granulation tissue. Further, $\mathrm{Mg} 2+$ dependent ATPase activity was found to be influenced by Ashwagandha. Ashwagandha also reduced the succinate dehydrogenase enzyme activity in the mitochondria of granulation tissue (Begum \& Sadique, 1987).

\section{Anti-inflammatory effect due to Withaferin:}

Withaferin $\mathrm{A}$ and 3-b-hydroxy-2,3-dihydrowithanolide $\mathrm{F}$ isolated from Withania somnifera show promising antibacterial, antitumoral, immunomodulating and anti-inflammatory properties (Budhiraja and Sudhir, 1987).

\section{Anti-arthritic effect:}

Ashwagandha is an analgesic that soothes nervous system from pain response (Twajj et al., 1989). The powerful anti-arthritic properties (Singh et al. 1984, 1986) of Ashwagandha are now widely accepted and documented; it is furthermore found to be effective as antipyretic as well as analgesic also.

Ashwagandha ( $1000 \mathrm{mg} / \mathrm{kg} /$ oral) produced significant analgesic activity for a rat experiencing heat analgesia induced by hot plate method. The peak analgesic effect of Ashwagandha was recorded as 78.03 percent at 2 nd hour of administration. The involvement of pain mediators; prostaglandin and 5-hydroxytryptamine in analgesic activity of Ashwagandha was studied by pretreatment with paracetamol $(100 \mathrm{mg} / \mathrm{kg}$, ip) and cyproheptadine $(10 \mathrm{mg} / \mathrm{kg}$, ip). The analgesic activity of Ashwagandha was potentiated significantly by cyproheptadine, however, paracetamol failed to exhibit any significant change in its activity, suggesting the involvement of serotonin, but not prostaglandins in the analgesic activity of Ashwagandha (Mazen et al., 1990).

\section{Discussion and Conclusion}

The available scientific data support the conclusion that Ashwagandha is a real potent regenerative tonic (Rasayana of Ayurveda), due to its multiple pharmacological actions like anti-stress, neuroprotective, antitumor, anti-arthritic, analgesic and anti-inflammatory etc. It is useful for different types of diseases like Parkinson, dementia, memory loss, stress induced diseases, malignoma and others.

Ashwagandha is used as a household remedy by Indians, who consider it as the best tonic for old people and children, and as aphrodisiac by young people. It is one of the best nervine tonics of Ayurveda, the most ancient system of Medical Sciences. Our clinical experience showed that besides the enumerated neurological conditions, brain strokes causing paralysis and neuronal deficit also improve in the long term treatment with Ashwagandha. We are also using it in all forms of cancer including prostate and lung cancers, especially in last stages, giving the patients lot of health benefits. We have some cases of lung cancer who have refused modern therapy and recovered clinically and radiologically with our therapy of Ashwagandha. (Singh N., 2010- unpublished data). In a recent seminar (Singh, 2005) on essential drug concept, it was projected as one of the six essential medicinal herbs.

Thus, the above findings clearly indicate that the traditional use of Ashwagandha has a logical and scientific basis. Large scale clinical studies are needed to prove the clinical efficacy of this herb, specially in stress related diseases, neuronal disorders and cancers.

\section{References}

1. Abbas, S.S. and Singh, N. (2006). Anti-stress Agents (Herbs) of Indian Origin - Herbal Drugs, A twenty first century perspective. Institute of Nuclear Medicine and Allied Sciences, Defence Research and Development Organization (DRDO), Govt. of India, Delhi, 578-591.

2. Abbas, S.S, Singh, V., Bhalla, M., and Singh, N. (2004). Clinical Study of Organic Ashwagandha in cases of Parkinsonism, Neuropathy, Paralysis and Uterine Tumours (Fibroids and other tumours) including Cutaneous Endodermal 
Carcinoma. Proc., National Seminar on "Eco-friendly Herbs of Ayurveda in Healthcare of Mankind: A Strategy for Scientific Evaluation an Uniform Standardization" - Lucknow, 81.

3. Abbas, S.S., Bhalla, M. and Singh, N. (2005). A clinical study of Organic Ashwagandha in some cases of uterine tumors (fibroids) and dermatofibrosarcoma. Proc. workshop on essential medicines, adverse drug reactions and therapeutic drug monitoring. Scientific Convention Centre, Lucknow, 143-144.

4. $\quad$ Abdel-Magied, E.M., Abdel-Rahman, H.A. and Harraz, F.M. (2001). The effect of aqueous extracts of Cynomorium coccineum and Withania somnifera on testicular development in immature Wistar rats. J. Ethnopharmacol., 75: 1-4.

5. $\quad$ Archana, R., Namasivayam, A (1999). Antistressor effect of Withania somnifera. J. Ethnopharmacol., 64: 91-93.

6. Atta-ur-Rahman, Samina-Abbas, Dur-e-Shahwar, Jamal, S.A., Choudhary, M.I. and Abbas. S. (1991). New withanolides from Withania spp.. Journal of Natural Products 56: 1000-1006.

7. Begum, V.H., Sadique, J. (1987). Effect Of Withania-Somnifera On Glycosaminoglycan Synthesis in CarrageeninInduced Air Pouch Granuloma. Biochemical Medicine and Metabolic Biology, 38(3): 272-277.

8. Bhandari, C.R. (1970). Ashwagandha (Withania somnifera) "Vanaushadhi Chandroday" (An Encyclopedia of Indian Herbs). Publisher: CS Series of Varanasi Vidyavilas Press, Varanasi, India, 1: 96-97.

9. $\quad$ Bhattacharya, S.K., Goel, R.K., Kaur, R., Ghosal, S. (1987). Anti - stress activity of Sitoindosides VII and VIII. New Acylsterylglucosides from Withania somnifera. Phytother. Res., 1: 32-37.

10. Bhattacharya, S.K, Kumar, A., and Ghosal, S. (1995). Effects of glycowithanolides from Withania somnifera on animal model of Alzheimer's disease and perturbed central cholinergic markers of cognition in rats. Phytother. Res., 9: 110 113.

11. Budhiraja, R.D. and Sudhir, S. (1987). Review of biological activity of withanolides. JSIR, 46: 488-491.

12. Changhadi Govardhan Sharma (1938) - Ashwagandharishta - Rastantra Sar Evam Sidhyaprayog Sangrah Krishna-Gopal Ayurveda Bhawan (Dharmarth Trust), Nagpur 743-744.

13. Charak Samhita 6000BC (1949). Charaka translation into English: Translator: Shree Gulabkunverba Ayurvedic Society, Jamnagar, India.

14. Dekosky, S. and Scheff, S.W. (1990). Synapse loss in frontal cortex biopsies in Alzheimer's disease: correlation with cognitive severity. Ann. Neurol., 27: 457-464.

15. Dickon T.C. and Vickers J.C. (2001). The morphological phenotype of b-amyloid plaques and associated neuritic changes in Alzheimer's disease. Neuroscience, 105: 99-107.

16. Dixit, K.S., Agarwal, A.K., Seth, P.K. and Singh, N. (1995). Effect of Withania somnifera, Panex ginseng and Cannabis indica and radio ligand binding with neurohumoral in the CNS, World Congress on Biotech. Dev. Med. Subs. Plants \& Marine Origin, King George Medical College, Lucknow (India), 141.

17. Ghosal S., Srivastava R.S., Bhattacharya S.K., Upadhyay S.N., Jaiswal A.K., Chattopadhyay U. (1989). Immunomodulatory and CNS effects of sitoindosides IX and X, two new glycowithanolides form Withania somnifera. Phytother. Res., 2: 201- 206.

18. Gunne L.M. and Andren P.E. (1993). An animal model for coexisting tardive dyskinesia and tardive parkinsonism: A glutamate hypothesis for tardive dyskinesia. Clin. Neuropharmacol., 16: 90-95.

19. Kalsi, R., Singh, N. and Gupta, G.P. (1987). Effects of stress and anti-stress drugs on succinate dehydrogenase enzyme (SDH) in rat brain (A possible role of SDH in stress adaptation phenomenon) Physiology of Human Performance, Defence Institute of Physiology and Allied Sciences, Defence Research and Development Organization (DRDO), Govt. of India, Delhi, 114-117.

20. Kritikar, K.R. and Basu, B.D. (1935). Withania somnifera, Indian medicinal plants, $2^{\text {nd }}$ Edition, IIIrd Vol., Lalit Mohan Basu, Allahabad, 1774-1776.

21. Kuboyam, A. T., Tohda, C., Komatsu, K. (2006). Withanoside IV and its active metabolite, sominone, attenuate A beta(25-35)-induced neurodegeneration. Eur J Neurosci., 23(6): 1417-1426.

22. Kulkarni, S.K., Sharma, A, Verma, A. and Ticku, M.K. (1993). GABA receptor mediated anticonvulsant action of Withania somnifera root extract. Indian Drugs, 30: 305-312.

23. Mazen, E.S., Pavelescu, M., Grigorescu, E. (1990). Contributions to the Pharmacodynamic Study of roots of Withania somnifera Dun Species Of Pakistani origin. Testing of Analgesic Activity of Dichloromethanic and Methanolic Extract from Withania-Somnifera Roots; Revista Medico-Chirurgicala Societatii de Medici si Naturalisti din Iasi 94 (3-4): 603605 .

24. Misra B. (2004). Ashwagandha - Bhavprakash Nigantu (Indian Materia Medica) - Chaukhambha Bharti Academy, Varanasi, 393-394.

25. Mishra, L.C., Singh, B.B., Dagenais, S. (2000). Scientific basis for the therapeutic use of Withania somnifera. (Ashwagandha): A review. Alternative Medicine Reviews 5: 334-46.

26. Nagashyana N, Sankarankutty P, Nampoothiri MRV, Moahan P, Mohan Kumar P. (2000). Association of L- dopa with recovery following Ayurvedic medication in Parkinson's disease. J. Neurol. Sci., 176: 1121-1127.

27. Sharma G.S. (1938). Ashwagandharishta - Rastantra Sar Evam Sidhyaprayog Sangrah - Krishna-Gopal Ayurveda Bhawan (Dharmarth Trust), Nagpur 743-744.

28. Sharma, P.V. (1999). Ashwagandha, Dravyaguna Vijana, Chaukhambha Viashwabharti, Varanasi, 763-765.

29. Singh, N., Agarwal, A.K., Lata, A. and Kohli, R.P. (1976). Evaluation of 'adaptogenic' properties of Withania somnifera. Proc. Indian Pharmacological Society, 17.

30. Singh, N., Agarwal, A.K., Lata, A. and Kohli, R.P. (1977). Experimental evaluation of 'adaptogenic' properties of Withania somnifera. XIIth Scientific Seminar on Indian Medicine, Institute of Medical Sciences. Varanasi. 4.

31. Singh, N. (1981). A new concept on the possible therapy of stress disease with 'Adaptogens' (Anti-stress drugs) of indigenous plant origin. Curr. Med. Prac., 25: 50-55.

32. Singh, N., Singh, S.P., Sinha, J.N., Shanker, K., and Kohli, R.P. (1982). Withania somnifera (Ashwagandha) A rejuvenator herbal drug which enhances survival during stress (An adaptogen). Int. J. Crude Drug Res., 3: 29-35. 
33. Singh, N., Singh, S.P., Nath, C., Kohli, R.P. and Bhargava, K.P. (1984). Anti-stress plants as anti-rheumatic agents, $5^{\text {th }}$ Sepal Congress of Rheumatology, Bangkok, 37.

34. Singh, N. (1986). A pharmaco-clinical evaluation of some Ayurvedic crude plant drugs as anti-stress agents and their usefulness in some stress diseases of man. Ann. Nat. Acad. Ind. Med., 2(1):14-26.

35. Singh, N., Singh, S.P., Dixit, K.S., Saxena, R.C. and Kohli, R.P. (1986a). A Placebo Controlled Clinical Trial of Cyprus rotundus, Withania somnifera and their Combination in cases of Rheumatoid Arthritis. Proc. International Seminar., 2: $18-21$.

36. Singh, N., Singh, S.P., Nath, R., Singh, D.R., Gupta, M.L., Kohli, R.P. and Bhargava, K.P. (1986b). Prevention or Urethane-induced lung adenomas by Withania somnifera (L.) Dunal in albino mice. Int. J. Crude Drug Res. 24(2): 99-100.

37. Singh, N. (1988). Effect of Withania somnifera and Panax ginseng on dopaminergic receptors in rat brain during stress. XXXVIth Annual Congress on Medicinal Plant Research (Freiburg), 28.

38. Singh, N. (1993a). Stress disease and herbal medicine, Inaugural issue of J. Biotech. Med. Plant Res., Lucknow, 2-

4.

39. Singh, N. (1993b). Effect of Anti-stress agents on receptor population in rat brain, Inaugural issue of J. Biotech. Med. Plant Res., Lucknow, 14.

40. Singh, N. and Misra, N. (1993). Experimental methods tools for assessment of anti-stress activity in medicinal plants. Journal of Biomedical Research., 12(182): 124-127.

41. Singh, N. (1995a). Stress disease and herbal medicine. World Congress on Biotech. Dev. Med. Subs. Plants \& Marine Origin, King George Medical College, Lucknow (India), 48.

42. Singh, N. (1995b). Anti-stress (Ayurvedic Plants) Ocimum sanctum (Tulsi) and Withania somnifera (Ashwagandha) in prevention and treatment of cardiovascular disorders. Proc. Vth W.C.C.N., China, 10.

43. Singh, N., Singh, V. and Abbas, S.S. (2003). Role of Adaptogens / Antistress agents of plant origin in health care \& stress diseases of man. Proc. $2^{\text {nd }}$ World Cong. Biotech. Dev. Herbal Med., Lucknow (India), 33.

44. Singh, N. (2005). Dilemma of Ayurvedic Herbal Medicines / Food Supplements Proc. workshop on Essential Medicines, Adverse Drug Reactions and Therapeutic Drug Monitoring, Dept. of Pharmacology \& Therapeutics, KGMU, Scientific Convention Centre, Lucknow, 35-41.

45. Singh, N. (2006). Ayurvedo-Herbal Medicines - The Need of the Time - Herbal Drugs, A twenty first century perspective. Institute of Nuclear Medicine and Allied Sciences, Defence Research and Development Organization (DRDO), Govt. of India, Delhi, 535-547.

46. Singh, N. (2008). Herbs - The Life of Man, Need Pharmaco-clinical Studies for their Scientific Validation Relevance of Modern Methods of Pharmacological Studies to Traditional Medicine, Department of Pharmacology \& Therapeutics, C.S.M. Medical University, Scientific Convention Centre, Lucknow, 37-43.

47. Singh, N. and Gilca, M. (2010). Herbal Medicine - Science embraces tradition - a new insight into the ancient Ayurveda, Lambert Academic Publishing (Germany), 51-67.

48. Singh, N., Hoette, Y. and Miller, R. (2010) - Tulsi 'The Mother Medicine of Nature' $2^{\text {nd }}$ Edition, International Institute of Herbal Medicine, Lucknow, 28-32.

49. $\quad$ Singh R.H., Udupa K.N. (1993) Clinical and experimental studies on rasayana drugs and rasayana therapy. Special Research Monograph, Central Council for Research in Ayurveda and Siddha (CCRAS), Ministry of Health and Family Welfare, New Delhi.

50. Singh, R.S. (1983). Ashwagandha, Vanaushadhi Nidharsika (Ayurvedic Pharmacopia), UP Sansthan, 30-31.

51. Singh, S.P., Singh, D.R., Gupta, M.L., Singh, N. and Kohli, R.P. (1979). An experimental evaluation of anti-tumor activity of Withania somnifera (Ashwagandha) and "Geriforte." XI Annual Conf. IPS. Ind. Jour. Pharmacol 11(1): 65.

52. Sumanran, V.N., Boddul, S. and Madhuri, D. (2007). Differential growth inhibitory effects of Withania somnifera root on CHO cells. Phytother. Res., 21: 1-4.

53. Twaij, H.A.A., Elisha, E.E. and Khalid, R.M., (1989). Analgesic studies on some Iraqi medicinal plants; International Journal of Crude Research 27: 109-112. 